\title{
Perirhinal Cortex Supports Delay Fear Conditioning to Rat Ultrasonic Social Signals
}

\author{
Derick H. Lindquist, ${ }^{1}$ Leonard E. Jarrard, ${ }^{2}$ and Thomas H. Brown ${ }^{1}$ \\ ${ }^{1}$ Department of Psychology, Yale University, New Haven, Connecticut 06520, and 2Department of Psychology, Washington and Lee University, Lexington, \\ Virginia 24450
}

\begin{abstract}
Auditory information can reach the lateral nucleus of the amygdala (LA) through a monosynaptic thalamic projection or a polysynaptic cortical route. The polymodal input from the perirhinal cortex (PR) is a major informational gateway to the LA and nearby structures. Pretraining PR lesions impair fear conditioning to a context, but there have been no reports that they cause deficits in delay conditioning to an auditory cue. The direct subcortical projection to the LA seems sufficient to support delay conditioning to a tone conditional stimulus (CS). We examined the effect of PR lesions on delay conditioning to two different tone conditional stimuli (4 and $22 \mathrm{kHz}$ tones; both $10 \mathrm{sec}$ duration) and two different rat ultrasonic vocalization (USV) conditional stimuli (10 sec of " $22 \mathrm{kHz}$ USVs"). The two USV conditional stimuli were multi-call segments that were recorded (digitized at $100 \mathrm{kHz}$ ) from two different rats. One USV CS was a continuous sequence of eight calls, and the other was a portion of a continuous sequence of six calls. PR lesions significantly impaired conditioning to both USV conditional stimuli and to the training context but had no significant effect on conditioning to either tone CS. The role of PR in fear conditioning appears not to be determined by whether the conditional stimuli serve as contexts or cues, but instead by the nature or complexity of the stimuli or stimulus configurations. These cue-specific effects of PR lesions are suggested to reflect differences in the stimulus features that are encoded in the two CS pathways to the LA.
\end{abstract}

Key words: fear conditioning; medial temporal lobe; perirhinal cortex; ultrasonic vocalization; amygdala; freezing

\section{Introduction}

Auditory information can reach the lateral nucleus of the amygdala (LA) either through a monosynaptic projection from the thalamus or through a polysynaptic cortical pathway (Romanski and LeDoux, 1992b; Mascagni et al., 1993). Delay conditioning to a simple auditory conditional stimulus (CS) can be supported entirely by the subcortical projection to the LA (Phillips and LeDoux, 1995; Bucci et al., 2000). More specifically, pretraining lesions of the perirhinal cortex (PR) are always reported to have no significant effect on delay fear conditioning to a tone CS (Romanski and LeDoux, 1992a,b; Campeau and Davis, 1995; Phillips and LeDoux, 1995; Bucci et al., 2000). The results have been less consistent regarding the effects of pretraining PR lesions on contextual conditioning. Whereas initial studies found no significant lesion effect (Romanski and LeDoux, 1992a; Phillips and LeDoux, 1995; Herzog and Otto, 1997, 1998), subsequent analyses of the effects of more complete PR lesions reported significant impairment in both context conditioning (Bucci et al., 2000) and context discrimination (Bucci et al., 2002).

These findings suggest parallels to the hippocampus (HC),

Received 0ct. 28, 2003; revised Jan. 23, 2004; accepted Feb. 17, 2004.

This work was supported by National Institutes of Health Research Grant MH058405. Predoctoral training support (D.H.L.) was furnished by National Research Service Award Grant MH64331. We thank Heather Williams for useful discussion, Sharon Furtak for comments on this manuscript, and Mamoon Allaf for technical assistance.

Correspondence should be addressed to Dr. Thomas H. Brown, Department of Psychology, Yale University, 2 Hillhouse Avenue, New Haven, CT 06520. E-mail: thomas.brown@yale.edu.

DOI:10.1523/JNEUROSCI.4839-03.2004

Copyright $\odot 2004$ Society for Neuroscience $\quad$ 0270-6474/04/243610-08\$15.00/0 which supports fear conditioning to contexts (Maren et al., 1997; Holland and Bouton, 1999) but seems not to be essential for delay conditioning to cues (Selden et al., 1991; White and Salinas, 2003). The reasonableness of this comparison stems partly from the fact that the PR ultimately serves as an important cortical input pathway to the $\mathrm{HC}$ and output pathway from the $\mathrm{HC}$ (Burwell et al., 1995; Naber et al., 1999; Shi and Cassell, 1999). An alternative perspective, which is consistent with a growing body of evidence (Buckley and Gaffan, 1998; Murray and Bussey, 1999; Brown and Aggleton, 2001; Burwell and Witter, 2002), is that the PR supports conditioning to or learning about complex stimuli or stimulus configurations.

This "complexity/configural hypothesis" implies that the role of the PR in delay conditioning depends on the stimulus properties of the cue. Whereas the subcortical projection to the LA can support conditioning to a tone CS, conditioning to sufficiently complex cues is predicted to require $\mathrm{PR}$ processing. To evaluate this hypothesis, we used rat ultrasonic vocalizations (USVs) as "complex conditional stimuli". USV conditional stimuli were 10 sec continuous segments of so-called " $22 \mathrm{kHz}$ USVs." These frequency-modulated (FM) social signals occur in a narrow bandwidth $(20-30 \mathrm{kHz})$ and consist of a string of "long calls" (each >200 msec) (van der Poel and Miczek, 1991; Brudzynski and Barnabi, 1993; Choi and Brown, 2003). Rats communicate extensively through USVs in two frequency bands: 20-30 and 40-70 kHz (Sewell, 1970; Sales and Pye, 1975).

Here, we evaluated the effects of pretraining PR lesions on conditioning to two different USV conditional stimuli that dif- 
fered along several dimensions. The results suggest that the role of the PR in fear conditioning is determined not by whether the conditional stimuli serve experimentally as contexts or cues, but rather by the nature or complexity of the stimuli or stimulus configurations.

\section{Materials and Methods \\ Experiment 1}

PR-lesioned and sham-operated rats were conditioned and tested using one of three $10 \mathrm{sec}$ conditional stimuli: a $4 \mathrm{kHz}$ tone, a $22 \mathrm{kHz}$ tone, or a segment from a prerecorded $22 \mathrm{kHz}$ USV. As a control for CS-specific pseudo-conditioning, an explicitly unpaired group was included using the same $22 \mathrm{kHz}$ USV. All animals received $1 \mathrm{~d}$ of fear conditioning and $1 \mathrm{~d}$ of cue and context testing.

Subjects. Experiment 1 used 45 male Sprague Dawley rats (Charles River, Kingston, NY), housed individually. Each rat had ad libitum access to food and water and was kept in a vivarium with a $12 \mathrm{hr}$ dark/light cycle. Rats were handled at least $2 \mathrm{~d}(3-5 \mathrm{~min} / \mathrm{d})$ before surgery. At the time of surgery, subjects weighed between 300 and $355 \mathrm{gm}$. All of the following procedures, including surgery and postoperative care, were in strict compliance with the Yale Animal Resource Center guidelines.

Design and power. Rats were assigned randomly to one of four conditioning groups and one of two surgical groups. Three of the conditioning groups received paired presentations of one of three $10 \mathrm{sec}$ auditory cues with a coterminating $(0.5 \mathrm{sec}, 1.0 \mathrm{~mA})$ foot shock unconditional stimulus (US): $22 \mathrm{kHz}$ USV CS $(n=10), 22 \mathrm{kHz}$ tone CS $(n=10)$, and a $4 \mathrm{kHz}$ tone CS $(n=11)$. The fourth group received explicitly unpaired presentations of the USV CS and US $(n=11)$. Half of the animals in each experimental group received PR lesions, and the other half a sham operation. All rats experienced $1 \mathrm{~d}$ of conditioning and $1 \mathrm{~d}$ of testing. Testing measured the freezing conditional response $(\mathrm{CR})$ to both the training context and the cue in counterbalanced order. Whereas context conditioning was tested in the original training chamber, a context shift was introduced when testing cue conditioning.

The sample size was chosen to yield power $(1-\beta)>0.8$ (Cohen, 1988) for the surgical effect on conditioning to the USV cue, the CS of primary interest and the only novel one. The power estimate assumed a large lesion effect ( $\geq 60 \%$ CR reduction) because subtle effects, which could be important in another context, would not furnish direct support for the complexity/configural hypothesis. As a point of reference, note that Bucci et al. (2000) reported PR lesions reduced context-elicited freezing by $\sim 50 \%$.

Surgery. Subjects were anesthetized with an intraperitoneal injection of ketamine $(90 \mathrm{mg} / \mathrm{kg})$ and xylazine $(10 \mathrm{mg} / \mathrm{kg})$. A petroleum-based ophthalmic ointment was applied to both eyes to prevent the cornea from drying out. Throughout the surgery, animals were kept on a heating pad (Braintree Scientific, Braintree, MA) to maintain body temperature. All surgical procedures were performed under aseptic conditions. Aspiration PR lesions were used because we found this permits more complete and selective damage than neurotoxin lesions (Jarrard et al., 2004).

After making an incision from the caudal portion of the eye to the front of the ear, the skin was retracted, and attachment of the muscle to the temporal bone was separated along the extent of the incision, with care being taken to avoid cutting the muscle. The muscle and skin were retracted ventrally to expose the suture where the temporal and parietal bones are joined by the posterior extent of the zygomatic arch. A hand drill was used to remove a small portion of the overlying bone, the dura was removed, and the underlying rhinal fissure was visualized. Subpial aspiration of the PR was performed using a curved and blunted 23 gauge hypodermic needle attached to a vacuum pressure pump. After surgery, the temporal muscle was put back in place, and the wound was sutured closed. Sham-operated animals were incised, the temporal muscle was retracted, and an opening was created in the skull, but the PR was not aspirated.

Ultrasonic audio recording and playback. Context-elicited conditional $\sim 22 \mathrm{kHz}$ USVs were recorded using a high-frequency condenser microphone (model 7012 for 2-40 kHz; ACO Pacific, Belmont, CA), amplified by a MA2 preamplifier [Tucker-Davis Technologies (TDT), Gainesville, $\mathrm{FL}$ ], and then digitized at $100 \mathrm{kHz}$ by an RP2.1 digital signal processor

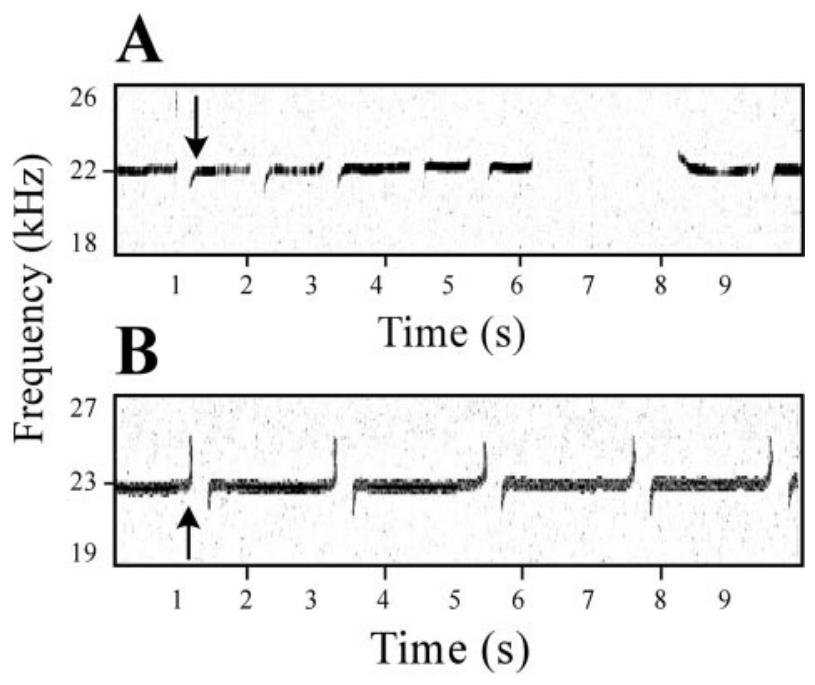

Figure 1. Digital recordings of fear-elicited $22 \mathrm{kHz}$ USVs. The top spectrogram $(A)$ is the USV CS used in experiment 1. The average call duration is $850 \mathrm{msec}$. The bottom spectrogram $(B)$ is the USV CS used in experiment 2. The average call duration is 1490 msec. The downwardpointing arrow in $A$ and the upward-pointing arrow in $B$ indicate the first instance of an upsweep at the beginning and end of the USV, respectively.

(TDT), as described previously (Choi and Brown, 2003). The digitized signals were stored on a computer running MATLAB, which was also used to play back the signal during cue conditioning. The output signal was sent to a high-frequency ES1 electrostatic loudspeaker (TDT) powered by an ED1 electrostatic loudspeaker driver (TDT). A heterodyne bat detector (Mini-3; Noldus Information Technologies, Wageningen, The Netherlands) transformed the high-frequency signals $(20-160 \mathrm{kHz})$ into the audible frequency range, furnishing an auditory signal for the experimenter (Brudzynski and Barnabi, 1993; Choi and Brown, 2003). The 22 $\mathrm{kHz}$ tone was generated using a $20 \mathrm{MHz}$ sweep/function generator (model 4040; B+K Precision, Yorba Linda, CA) and was presented through the same electrostatic loudspeaker and driver used for the USV CS.

The two USV conditional stimuli shown in Figure 1 were continuous $10 \mathrm{sec}$ segments of conditional responses to an aversive context that were recorded from two different rats (cf. Sewell, 1970; Sales and Pye, 1975; Blanchard et al., 1991; van der Poel and Miczek, 1991; Brudzynski and Barnabi, 1993; Choi and Brown, 2003). The peak output intensity for each USV CS was determined using an Ultraprobe 9000 (UE Systems, Elmsford, NY). The bandwidth of the Ultraprobe 9000 decibel meter is $20-100 \mathrm{kHz}$, recording $\pm 5 \mathrm{kHz}$ from the frequency set manually by the experimenter. The reported intensities are traceable to the standards enacted by the National Institute of Standards and Technologies. The first USV CS (Fig. $1 \mathrm{~A}$ ) was a $62 \mathrm{~dB}$ segment that contained eight individual calls. The mean call duration was $850 \mathrm{msec}$, and the coefficient of variation of the durations was 0.3 (cf. van der Poel and Miczek, 1991; Brudzynski and Barnabi, 1993; Choi and Brown, 2003). When identically measured, the intensity of the $22 \mathrm{kHz}$ tone was $67 \mathrm{~dB}$. As in our previous studies (Choi et al., 2001; Lindquist and Brown, 2004), the intensity of the $4 \mathrm{kHz}$ tone was $75 \mathrm{~dB}$, measured using the $\mathrm{C}$ scale of a digital sound meter (model 33-2055; range, 32-10,000 Hz; Realistic, Fort Worth, TX).

Conditioning and testing chambers. Four different chambers were used for conditioning and testing, each of which was equipped with an infrared light source and video camera (CB-21; Circuit Specialists, Mesa, AZ) to observe and record behavior during the experiment. The video signal from each chamber was fed to video recorders for offline analysis of freezing. The $10 \mathrm{sec}$ auditory CS was delivered by a speaker mounted near the conditioning chamber. The coterminating, grid-shock US (500 msec, $1.0 \mathrm{~mA}$ ) was produced by a small-animal shock generator (E13-14; Coulbourn Instruments, Allentown, PA). The root mean square current was measured conventionally (Choi et al., 2001) from the voltage across the smaller of two resistors ( 1 and $100 \mathrm{k} \Omega$ ) connected in series between adjacent grid bars. The conditioning chambers each had a standard grid 


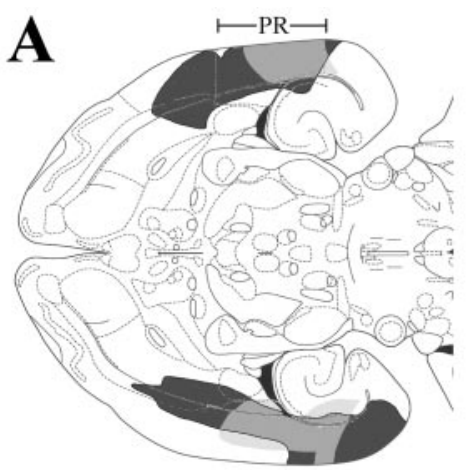

Plate 98

Interaural $2.40 \mathrm{~mm}$

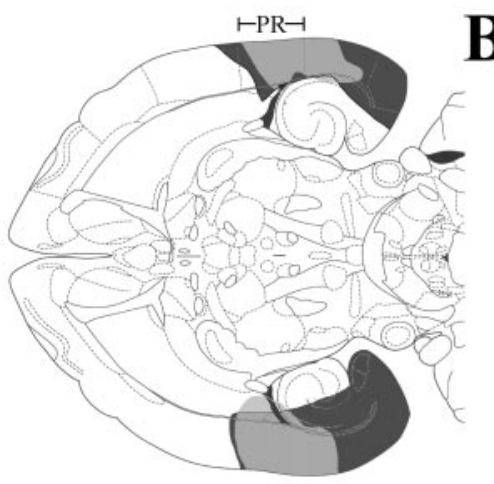

Plate 100

Interaural $2.90 \mathrm{~mm}$

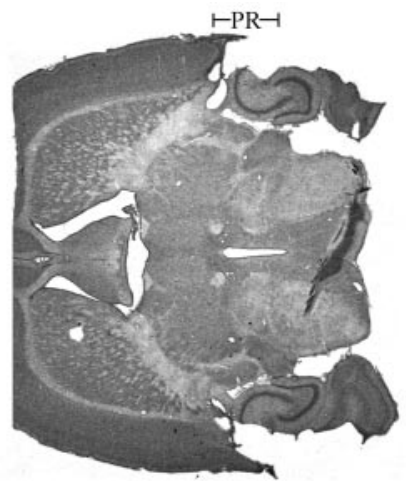

Plate 100

Interaural $2.90 \mathrm{~mm}$

Figure 2. Composite histological reconstructions and a photomicrograph showing PR damage in experiment 1.A, PR lesions drawn onto plates 98 and 100 from Paxinos and Watson (1998). Gray and black areas indicate, respectively, the smallest and largest lesion. The rostrocaudal extent of the PR, indicated above both plates, follows the coordinates of Paxinos and Watson (1998). The region of the PR in plate 98 ranges from -2.4 to $6.0 \mathrm{~mm}$, whereas plate 100 ranges from -2.7 to $5.0 \mathrm{~mm}$, relative to bregma. The numbers at the bottom of each figure indicate the distance in millimeters above the interaural line. $B$, Photomicrograph of a stained section, corresponding to plate 100 , showing a representative PR lesion. The rostrocaudal extent of the PR is indicated by the bar at the top of the figure.

floor consisting of parallel steel rods $(5 \mathrm{~mm}$ diameter and $15 \mathrm{~mm}$ spacing). The interior dimensions were 22 (length) $\times 28$ (width) $\times 42$ (height) centimeters. The chambers were housed in sound-attenuating cubicles (Choi et al., 2001; Lindquist and Brown, 2004). Cue testing was done in a context that differed in three respects: the chamber was cleaned with a different odorant; the standard grid floor was replaced with a solid one; and a light bulb $(\sim 25$ lux $)$ at the side of the chamber was illuminated.

Conditioning and testing procedures. Fear conditioning began 10-14 d after surgery. Each chamber was cleaned using a vinegar/water (1:3) solution or Windex before placing the rat in the chamber. After placement into the conditioning chamber, each rat was given a $5 \mathrm{~min}$ baseline period to explore the environment. All subjects then received 10 pairings of the $10 \mathrm{sec}$ auditory CS and the coterminating $500 \mathrm{msec}$ US ( $9.5 \mathrm{sec}$ interstimulus interval). Ten pairings were deemed sufficient to reveal even slow fear conditioning (cf. Maren, 1999). The intertrial interval (ITI) was chosen from a preselected uniform distribution (30 sec bins) ranging from 180 to $300 \mathrm{sec}$ (mean, 240). The unpaired control group was presented with the same USV CS and grid-shock US, but in a pseudorandom, explicitly unpaired order, such that no more than two conditional stimuli or unconditional stimuli occurred consecutively. The ITI during the unpaired conditioning session was selected randomly from a uniform distribution (15 sec bins) ranging from 60 to $180 \mathrm{sec}$ (mean, 120 ). Rats assigned to the explicitly unpaired group received the same number and density of CS and US presentations as in the other three groups.

Responses to the cue and the conditioning context were tested on the following day. To assess conditioning to the context, the rat was placed into the original conditioning chamber for $11 \mathrm{~min}$. After a $2 \mathrm{~min}$ baseline period, freezing was measured for $8 \mathrm{~min}$. Similarly, after a $2 \mathrm{~min}$ baseline period, cue-specific responding was assessed in a shifted context for an 8 min period, with freezing quantified during the $60 \mathrm{sec}$ interval just before CS onset, during the 6 min CS presentation, and then for another $60 \mathrm{sec}$ after CS offset. Freezing was defined as cessation of all movement, except that required for respiration (Blanchard and Blanchard, 1969; Fanselow, 1997). The total duration of freezing was measured during the $8 \mathrm{~min}$ interval for context testing and for each of the three stages of cue testing (60 sec pre-CS, $6 \mathrm{~min}$ during the CS, and $60 \mathrm{sec}$ post-CS) and then converted to a percentage score (time freezing/total interval time). All freezing data were analyzed by the experimenter and a blind assistant, resulting in an interobserver correlation of $r=0.94$ (cf. Lindquist and Brown, 2004). The pattern of statistical significance was identical for both, although only freezing scores collected by the blind assistant were used for data analysis.

Histology. After behavioral testing, animals were deeply anesthetized with Nembutal and halothane and perfused with physiologically buffered saline, followed by a $10 \%$ formalin solution. The brain was removed from the skull and stored in 10\% formalin for a minimum of $24 \mathrm{hr}$, followed by storage in a $30 \%$ sucrose solution for $3-4 \mathrm{~d}$. The brain was sectioned at $60 \mu \mathrm{m}$ with a freezing-stage microtome and mounted on gelatin-coated slides. Lesions were sectioned in the horizontal plane to be in register with studies of the cellular neuroanatomy and neurophysiology of the PR (Moyer and Brown, 1998; Faulkner and Brown, 1999; Beggs et al., 2000; Furtak et al., 2001; McGann et al., 2001; Moyer et al., 2002).

Microscopic examination (Zeiss Axioskop, $2.5 \times$ objective; Carl Zeiss Microimaging, Thornwood, NY) of each thionine-stained section identified the extent of each lesion, which was then traced, using a camera lucida, onto appropriately matched plates from the brain atlas of Paxinos and Watson (1998). The percentage of damage to the affected structures [PR, LA, HC, and entorhinal cortex (EC)] was estimated, based on the camera lucida tracings, from the ratio of the weight of each excised tracing of a damaged area to the weight of a matched tracing of the entire structure of interest. Plate 98 corresponds to regions of the PR ventral to the rhinal fissure (region 35), whereas plate 100 corresponds approximately to the level of the fissure or just above it (region 35, 36, or both). Portions of plates 98 and 100, defined here as the PR (Fig. 2), are within the region identified as the PR by Burwell et al. (1995, 2001). Plate 101 was not used because its relationship to the coronal sections of Burwell et al. $(1995,1998)$ is uncertain. Photomicrographs of selected sections were taken using the Zeiss Axiophot module.

\section{Experiment 2}

In the second experiment, a partial replication of the first one, the USV CS was a $10 \mathrm{sec}$ USV segment recorded from a different rat (Fig. $1 B$ ). The purpose was to assure that the results generalized to a second $22 \mathrm{kHz}$ USV CS that was specifically chosen to differ along several dimensions. Recall that the first $10 \mathrm{sec}$ USV CS (Fig. $1 \mathrm{~A}$ ) was a $62 \mathrm{~dB}$, eight-call sequence that contained a $\sim 2 \mathrm{sec}$ gap between two of the calls, the mean call duration was $850 \mathrm{msec}$, and the coefficient of variation of the call durations was 0.3 . The second $10 \mathrm{sec}$ USV CS (Fig. $1 \mathrm{~B}$ ) included five complete calls plus the start of a sixth one; none of the intercall intervals exceeded $300 \mathrm{msec}$, the mean call duration was $1490 \mathrm{msec}$, the coefficient of variation of the call durations was 0.5 , and the peak intensity was $54 \mathrm{~dB}$. The two USV conditional stimuli also differed in regard to the presence and frequency range of "upsweeps" at the start or end of each call (Fig. 1, arrows).

Subjects, surgeries, and behavioral training. Sprague Dawley male rats (295-360 gm) received either PR lesions $(n=8)$ or sham operations $(n=$ 6) as before. Other than the USV CS, the experimental procedures were identical to those in experiment 1 . Rats were conditioned with 10 pairings of a $10 \mathrm{sec}$ USV CS and a coterminating $500 \mathrm{msec}, 1.0 \mathrm{~mA}$ foot shock 

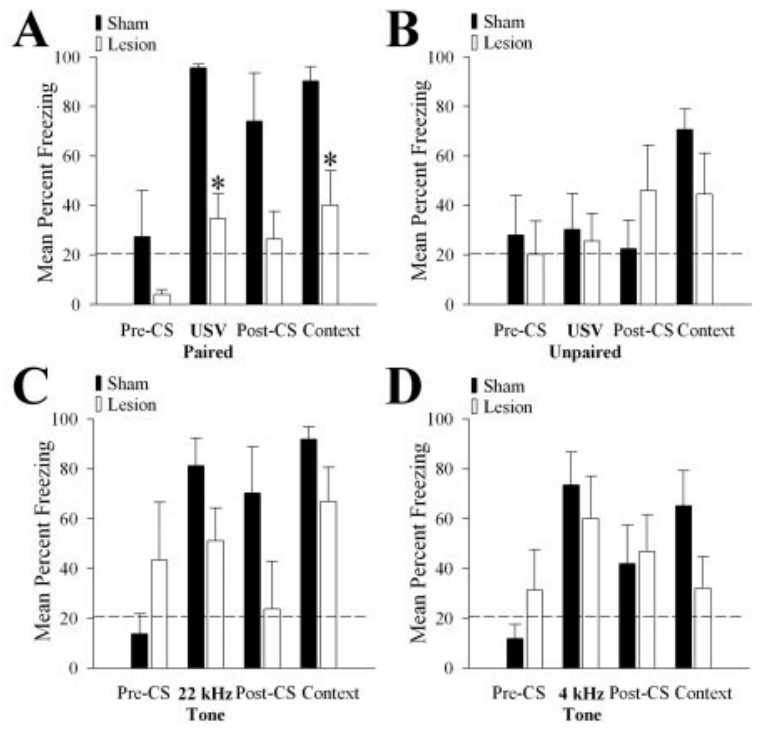

Figure 3. Freezing to the cue and context in each of the four groups in experiment 1. The dashed horizontal line is the mean level of freezing during the pre-CS period averaged across the four experimental groups (20.2\%), whereas asterisks denote significant differences between the sham-operated control and lesioned animals (see Results for details). $A$, Freezing to the paired USV cue and the training context. $B$, Freezing to the unpaired USV cue and the training context. C, Freezing to the paired $22 \mathrm{kHz}$ tone and the training context. D, Freezing to the $4 \mathrm{kHz}$ tone and the training context.

US. Twenty-four hours later, each subject was tested, in counterbalanced order, to the conditioning context and cue. The sample size was again selected (this time based on more accurate information from the first experiment) to yield $1-\beta>0.8$ for conditioning to the USV CS.

\section{Results}

\section{Experiment 1}

Subjects and histology

A total of 45 rats underwent surgery, 42 of which were used in data analysis. Three animals were dropped because of misplaced lesions or insufficient PR damage ( $<60 \%$ damage). Among lesioned animals $(n=20)$, PR damage included both areas 35 and 36 (Fig. 2). The mean \pm SE percentage of PR that was damaged, estimated from plates 98 and 100, was $82 \pm 3 \%$. The median amount of damage was $83 \%$, and the coefficient of variation was 0.1 . The smallest (gray) and largest (black) lesions from horizontal sections are shown mapped onto plates 98 and 100 (Fig. 2A). Figure $2 B$ is a photomicrograph of a thionine-stained section, at the level of plate 100, in which $77 \%$ of the PR was damaged. ANOVA revealed no significant difference in PR lesion size among the four conditioning groups $\left(F_{(3,16)}=0.72 ; p=0.56\right)$. The larger lesions also damaged surrounding structures. On the same two plates used for PR estimates, there was damage to $9 \pm$ $2 \%$ of the $\mathrm{HC}, 39 \pm 4 \%$ of the EC, and $53 \pm 4 \%$ of the LA.

\section{Freezing to the USV cue}

Sham-operated control rats showed almost maximal freezing to the USV CS (Fig. 3A, USV Paired, solid bar), with a mean \pm SE value of $95.7 \pm 1.4 \%$ (cf. Furtak et al., 2003). In Figure 3 and others, the dashed horizontal lines give a useful visual reference point or baseline for evaluating treatment effects. This baseline is the average level of freezing among all animals $(N=42)$ during the pre-CS period $(20.2 \%)$, further analyzed below. In control rats, the mean level of freezing to the USV CS was more than 50 SEs above this baseline level. In stark contrast, the mean level of freezing to the USV CS in PR-lesioned rats was $34.7 \pm 10.0 \%,<2$
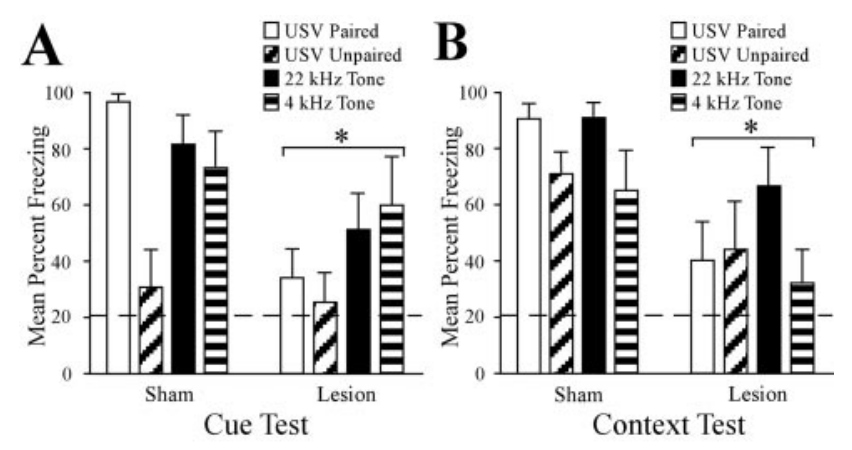

Figure 4. Freezing to the cue and context in the combined groups from experiment 1 . The broken horizontal line is the mean level of freezing during the pre-CS period (20.2\%), as in Figure 3 , and the asterisk denotes a significant overall effect of surgery. $A$, In the cue test, all three paired conditional stimuli elicited robust freezing in control rats (Sham; three tall bars), but not the unpaired USV CS (Sham; short bar). Freezing to the two tone conditional stimuli (Lesion; two tall bars) was not blocked by PR lesions, whereas lesions abolished freezing to the paired USV CS (Lesion; unfilled short bar), comparable with the level seen when the USV CS was unpaired (Lesion; other short bar). $B$, In the context text, all four groups of control animals showed robust freezing, which was significantly attenuated in the PR rats.

SEs above the baseline. PR lesions reduced freezing to the USV CS by $64 \%$ (calculated as [(mean percentage freezing in lesioned rats $) /($ mean percentage freezing in control rats $)-1] \times 100 \%)$. These large group differences were statistically significant $(t(8)=$ $6.06 ; p<0.001 ; 1-\beta>0.99)$. The surgical effect on freezing was insignificant during both the pre-CS period $(p=0.25)$ and post-CS period ( $p=0.07$ ).

The sham-operated control rats that were conditioned to the USV CS showed robust context conditioning (Fig. 3A, Context, filled bar). The mean \pm SE level of freezing to the context was $90.3 \pm 5.8 \%, 12$ SEs above the pre-CS baseline period (20.2\%). In contrast, context conditioning in PR-lesioned rats was only $40.1 \pm 14.0 \%$ (Fig. 3A, Context, open bar), $<2$ SEs above the baseline level. PR lesions reduced freezing to the context by $56 \%$. The surgical effect on freezing to the context was statistically significant $(t(8)=3.31 ; p<0.05 ; 1-\beta>0.84)$.

Little freezing was elicited in response to the USV cue in the explicitly unpaired group (Fig. 3B, USV Unpaired) in either the sham-operated $(30.2 \pm 14.6 \%)$ or PR-lesioned $(25.6 \pm 11.1 \%)$ rats. In both groups, the mean percentage of freezing was within $1 \mathrm{SE}$ of the baseline level, and there was no significant effect of surgery $(p=0.81)$. Combining the sham and lesion unpaired groups $(n=11)$, the mean percentage of freezing, just before the CS onset $(24.2 \pm 14.6 \%)$, was almost identical to the mean level during its presence $(27.9 \pm 12.9 \%)$, differences that were not significant (paired $t(10)=0.24 ; p=0.81$ ). The USV CS clearly does not elicit significant freezing unless it is paired with the US (see Fig. $4 A$ and associated discussion). There were no significant surgical effects on freezing during the pre-CS $(p=0.73)$ or post-CS ( $p=0.29)$ periods. Although unresponsive to the unpaired cue, the sham-operated rats did exhibit normal conditioning to the context $(70.7 \pm 8.1 \%)$. The level of freezing to the training context was only $44.6 \pm 16.4 \%$ in PR-lesioned rats, but the effect of surgery was not significant $(p=0.17 ; 1-\beta>0.26)$ (Fig. 6B; see Discussion).

Freezing to the tone cues and context

Both sham- and PR-lesioned rats showed obvious freezing to the $22 \mathrm{kHz}$ tone CS (Fig. 3C, $22 \mathrm{kHz}$ tone). The mean \pm SE percentage of freezing was $81.2 \pm 11.1 \%$ in control rats and $51.2 \pm 13.1 \%$ 
in lesioned animals, differences that were not statistically significant $(p=0.12)$. There was also no significant surgical effect on freezing during either the pre-CS $(p=0.26)$ or post-CS $(p=0.12)$ periods. Control rats froze more to the training context $(91.8 \pm 5.0 \%)$ than lesioned rats $(66.8 \pm 13.7 \%)$, but the surgical effect was not significant $(p=0.12 ; 1-\beta>0.32)$ (Figs. $4 B, 6 B$; see Discussion).

The $4 \mathrm{kHz}$ tone CS supported freezing in both sham-operated and PR-lesioned rats (Fig. $3 D, 4 \mathrm{kHz}$ tone). The mean $\pm \mathrm{SE}$ percentage of freezing was $73.5 \pm 13.1 \%$ in control rats and $60.1 \pm 16.9 \%$ in lesioned rats, resulting in a nonsignificant surgical effect ( $p=0.54)$. The surgical effect was also insignificant for freezing during the pre-CS $(p=0.25)$ and post-CS $(p=0.83)$ periods. Context conditioning in the control rats $(65.1 \pm 14.2 \%)$ (Fig. $3 D$, Context) was normal, although the mean level of freezing in lesioned rats $(32.1 \pm 12.5 \%)$ was only $1 \mathrm{SE}$ above baseline. The effect of surgery on freezing to the context was not significant $(p=0.12 ; 1-\beta>0.32$ ) (Figs. $4 B, 6 B$; see Discussion).

\section{Freezing in the combined groups}

To furnish an overview and summary of the four experimental groups, and also to increase the statistical power $(1-\beta)$ for detecting the reported PR lesion effect on context conditioning (cf. Bucci et al., 2000), the data for cue and context conditioning were combined (Fig. 4) $(N=42)$. The dashed horizontal line in Figure 4 is the same reference point used in Figure $3(20.2 \%$ freezing). After CS-US pairings, all three auditory cues elicited robust freezing in sham-operated control rats (Fig. 4A, Sham, three tall bars). The overall mean \pm SE level of freezing to the three paired conditional stimuli $(n=16)$ was $82.9 \pm 6.1 \%$. When the USV CS was not paired with the US (Fig. $4 A$, Sham, short bar), the level of freezing in its presence $(30.2 \pm 14.6 \%)$ was close to the overall pre-CS baseline level (20.2\%; dashed horizontal line) and also similar to the level in PR-lesioned rats that received either paired $(34.7 \pm 10.0 \%)$ or unpaired $(25.6 \pm 11.1 \%)$ presentations of this cue (Fig. $4 A$, Lesion, two short bars).

Whereas PR lesions significantly impaired conditioning to the USV CS (Fig. 4A, Lesion, short bar), they clearly did not prevent conditioning to the two tone conditional stimuli (Fig. 4A, Lesion, two tall bars). The mean \pm SE level of freezing to the two tone conditional stimuli was $77.0 \pm 8.4 \%$ among controls and $55.6 \pm$ $10.2 \%$ among lesioned animals, differences that were not significant $(p=0.13)$. The overall effect of surgery $\left(F_{(1,34)}=9.54 ; p<\right.$ $0.01)$ and experimental group $\left(F_{(3,34)}=4.84 ; p<0.01\right)$ were statistically significant, but the surgery $\times$ group interaction was not $(p=0.14)$.

The mean \pm SE level of freezing to the context among control animals $(n=22)$ was $79.5 \pm 8.3 \%$ (Fig. $4 B$, Sham). Among PR-lesioned rats $(n=20)$, the overall level of freezing was reduced by almost half to $45.9 \pm 14.2 \%$ (Fig. $4 B$, Lesion), an effect that was statistically significant $\left(F_{(1,34)}=15.74 ; p<\right.$ $0.001 ; 1-\beta>0.95)$. The lesion effect on conditioning to the context was comparable with that reported previously (Bucci

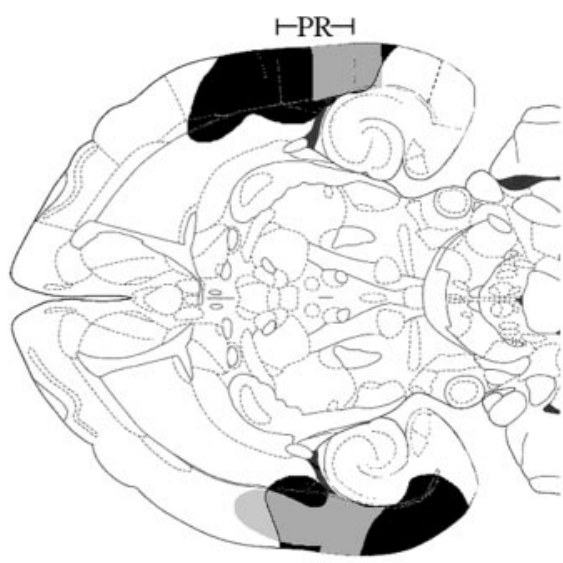

Plate 98

Interaural $2.40 \mathrm{~mm}$
Plate 100

Interaural $2.90 \mathrm{~mm}$ USV CS (also see Fig. $6 B$ and associated discussion).

\section{Experiment 2}

Subjects and histology

A total of 14 rats underwent surgery, 10 of which were used in data analysis. Three animals were eliminated because of misplaced lesions or insufficient PR damage $(<60 \%)$, and one animal was dropped because of a corrupted video signal during testing. Among the PR-lesioned animals $(n=5)$, the smallest (gray) and largest (black) lesions from horizontal sections are shown mapped onto plates 98 and 100 (Fig. 5). The mean \pm SE percentage of the PR that was damaged, estimated from plates 98 and 100 , was $76 \pm 6 \%$; the median amount of damage was $70 \%$, and the coefficient of variation was 0.2 . On the same two plates used for PR estimates, $2 \pm 1 \%$ of the $\mathrm{HC}, 17 \pm 5 \%$ of the $\mathrm{EC}$, and $55 \pm$ $10 \%$ of the LA were also damaged.

\section{Freezing to the USV cue and context}

The mean \pm SE level of freezing (Fig. 6A) in sham-operated control rats to the USV CS was $97.4 \pm 2.0 \%$, whereas the level of freezing to the USV CS in PR-lesioned rats was diminished to $26.1 \pm 9.9 \%$; differences that were statistically significant $(t(8)=$ $7.08 ; p<0.001 ; 1-\beta>0.99)$. Freezing to the context in shamoperated control rats was $96.9 \pm 2.6 \%$, compared with $57.9 \pm$ $8.1 \%$ in PR-lesioned rats, differences that were also statistically significant $(t(8)=4.58 ; p<0.01 ; 1-\beta=0.98)$. As in the first experiment, the lesion effect size was larger for conditioning to the USV cue than for conditioning to the context. Not shown in Figure $6 \mathrm{~A}$ are the facts that sham-operated control rats froze $40.0 \pm 21.5 \%$ during the pre-CS period and $74.7 \pm 19.0 \%$ during the post-CS period, whereas PR-lesioned rats froze $13.0 \pm 9.1 \%$ and $3.3 \pm 2.9 \%$, respectively, during these periods. There was a significant effect of surgery on freezing during the post-CS period $(t(8)=3.71 ; p<0.01)$ but not during the pre-CS period $(p=$ $0.28)$. The dashed line in Figure $6 A$ shows the baseline freezing level in this group of animals $(26.5 \% ; n=10)$.

\section{Experiments 1 and 2 combined}

To appraise better the relative effects of $\mathrm{PR}$ lesions on conditioning to the USV CS versus the training context, the results from experiment 2 were combined with the comparable groups from 

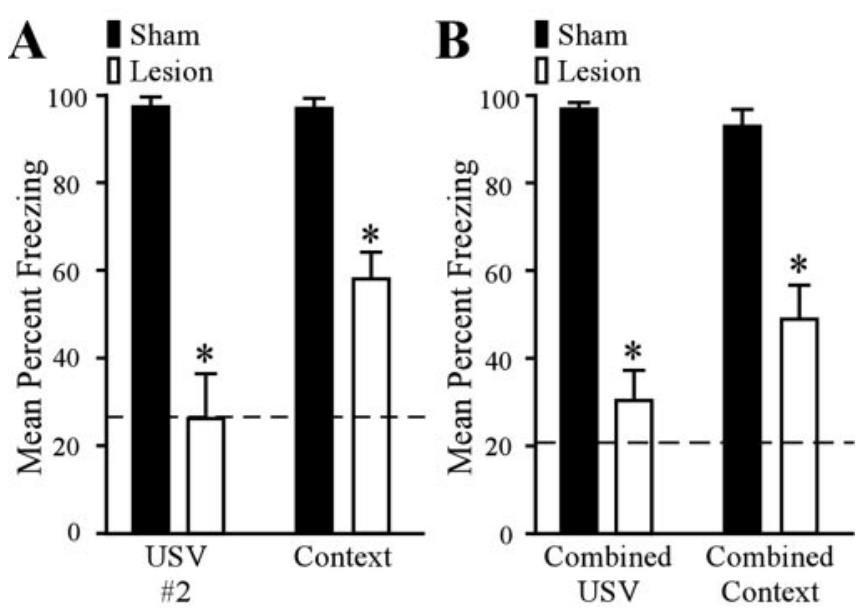

Figure 6. Replication and summary of PR lesion effects on conditioning to an USV cue and to the conditioning context. $A$, Freezing to USV \#2 and to the conditioning context in experiment 2 . The dashed horizontal line is the mean level of freezing during the pre-CS period (26.5\%). Asterisks denote significant differences between the sham-operated control and PR-lesioned animals. Freezing during the pre-CS and post-CS periods are not shown in this case (see Results for values). B, Freezing to both USV cues and to the conditioning context in the combined results from experiments 1 and 2 . The dashed horizontal line is the mean level of freezing during the combined pre-CS period (21.1\%). Asterisks denote a significant lesion effect.

experiment 1 . Before combining the results $(n=20)$, the data were examined for heterogeneity of variance. No evidence of inhomogeniety was found for cue conditioning (Levene $(18)=$ $0.60 ; p=0.45)$ or context conditioning $($ Levene $(18)=0.55 ; p=$ $0.47)$. The combined results are shown in Figure $6 B$, where the dashed horizontal line is the pre-CS baseline level of freezing (21.1\% in this case), as in previous figures.

The lesion reduced freezing to the USV cue by an impressive $69 \%$. The mean \pm SE level of freezing in sham-operated rats $(n=$ 10 ) to the USV cues was $96.6 \pm 1.2 \%$, whereas the level of freezing in PR-lesioned rats $(n=10)$ was $30.4 \pm 6.8 \%$, slightly $>1 \mathrm{SE}$ above the pre-CS baseline $(21.1 \%)$. This large lesion effect on conditioning to the USV CS was significant $(t(18)=9.63 ; p<$ $0.001)$, denoted by the asterisk in Figure $6 B$. In the same 10 lesioned animals, freezing to the context was reduced by $48 \%$. The sham-operated controls $(n=10)$ froze $93.6 \pm 3.2 \%$ to the conditioning context, within three percentage points of the level of freezing to the USV CS. Freezing to the context was reduced to $49.0 \pm 8.2 \%$ in the lesioned rats $(n=10)$, an effect that was significant $(t(18)=5.08 ; p<0.001)$, denoted by the asterisk in Figure $6 B$.

Although the lesion had a large and significant effect on freezing to the context, the level remained $>3$ SEs above baseline freezing $(21.1 \%)$ (Fig. $6 B$ ). Thus, the lesion greatly attenuated but did not completely abolish freezing to the context. Differences between the lesion effects on context versus cue conditioning were not statistically significant $(t(9)=1.89 ; p=0.09)$. The statistical power was insufficient (see Materials and Methods) to detect small differences (19\% in this case), among the lesioned rats $(n=10)$, between freezing to the USV CS $(30 \%)$ and freezing to the context (49\%).

\section{Discussion}

Pretraining PR lesions significantly impaired delay fear conditioning to both $22 \mathrm{kHz}$ USV conditional stimuli (Figs. 3A, 6), while having no significant effect on conditioning to either the 4 or the $22 \mathrm{kHz}$ tone CS (Figs. 3C,D, 4A). This last finding comple- ments and extends to higher frequencies the results of previous studies (Romanski and LeDoux, 1992a,b; Campeau and Davis, 1995; Phillips and LeDoux, 1995; Bucci et al., 2000) that reported cortical processing not to be required for delay conditioning to a tone CS. The elicitation of freezing by the USV cue was strictly conditional on previous pairings with the US (Figs. $3 A, B, 4 A$ ), indicating that responding to this novel CS does not reflect CSspecific pseudo-conditioning. The overall results (Figs. 3, 4A, 6) are consistent with the hypothesis (Romanski and LeDoux, 1992b) that delay conditioning to tone cues does not require cortical processing but can be acquired via the direct subcortical CS pathway to the amygdala.

\section{Previous studies of pretraining PR lesion effects on cue conditioning}

In all previous studies (Romanski and LeDoux, 1992a,b; Campeau and Davis, 1995; Phillips and LeDoux, 1995; Bucci et al., 2000), pretraining PR lesions have been reported to have no significant effect on subsequent CR expression to an acoustic cue. In some of these studies (Romanski and LeDoux, 1992a,b; Phillips and LeDoux, 1995), the lesions were not specifically targeted to the $\mathrm{PR}$, but rather PR damage was included as part of a much larger lesion. In another study, which reported pretraining PR damage not to impair conditioning to an acoustic cue (Campeau and Davis, 1995), the lesion was actually centered in the agranular insular cortex (AIC), immediately anterior to the PR. In contrast, damage to the AIC and anterior PR has been reported to impair conditioning to odorant cues (Herzog and Otto, 1997, 1998). In this case, the lesion presumably disrupted the CS pathway, because the AIC and anterior PR receive direct olfactory input and are reciprocally connected with the LA (Otto et al., 2000).

\section{Mnemonic effects of PR lesion size and of extra-PR damage}

Bucci et al. (2000) noted that PR lesions must be relatively complete to impair context conditioning, speculating that incomplete lesions might account for two previous failures (Herzog and Otto, 1997, 1998) to find a significant effect of pretraining PR lesions on context conditioning (but see Phillips and LeDoux, $1995)$. In the present study, the estimated mean \pm SE percentage of the PR that was damaged in all lesioned rats $(n=25)$ was $81 \pm$ $2 \%$. In agreement with Bucci et al. (2000), we found a significant lesion effect on context conditioning (Fig. 4B, 6B). Furthermore, the magnitude of the lesion effect in the present study $(48 \% \mathrm{CR}$ reduction; $n=20$ ) (Fig. $6 B$ ) was comparable with the value reported by Burwell and coworkers (50\% CR reduction, $n=22$ ) (Bucci et al., 2000, their Fig. 5B).

Although successful in removing most of the $\mathrm{PR}$, the aspirative surgical procedure also damaged the $\mathrm{HC}(7 \pm 2 \%$; $n=25)$, EC $(35 \pm 4 \% ; n=25)$, and LA $(53 \pm 4 \% ; n=25)$. In considering these percentages, it is important to remember they refer only to stereotaxic plates 98 and 100 . Whereas these two plates furnish reasonable estimates of $\mathrm{PR}$ and $\mathrm{EC}$ damage, they overestimate the total percentage damage to both the HC and LA. Despite substantial damage at the level of plates 98 and 100, the LA obviously did remain functional in the lesioned animals, because these rats showed robust conditioning to the tone CSs. Neither the EC nor the $\mathrm{HC}$ has been implicated in delay conditioning to a tone cue, although nothing is known about their possible role(s) in conditioning to these FM social signals (van der Poel and Miczek, 1991).

To evaluate further this extra-PR damage, we computed the partial correlations between lesion size and cue conditioning. The partial correlation between LA lesion size and cue conditioning, 
controlling for PR, HC, and EC damage, was statistically insignificant $(r=-0.20 ; p=0.37)$. Similarly, the partial correlations between cue conditioning and EC lesion size $(r=0.12 ; p=0.58)$ and HC lesion size $(r=0.01 ; p=0.98)$ were also insignificant. In contrast, the partial correlation between PR damage and cue conditioning, now controlling for LA, EC and HC lesion size, was significant $(r=0.48 ; p<0.05)$.

Two previous studies were specifically designed to dissociate the mnemonic functions of EC versus PR circuits. A double dissociation was reported in the rat, where pretraining lesions of the $\mathrm{PR}$ and the adjacent postrhinal cortex (POR), but not the EC, impaired nonspatial working memory, whereas damage to the EC, but not the PR and POR, produced deficits in spatial reference memory (Jarrard et al., 2004). Damage to the PR, but not the $\mathrm{EC}$, was also reported to interfere with working memory in rhesus monkeys (Meunier et al., 1993). On a visual delayed nonmatching-to-sample task, PR ablation impaired working memory almost as severely as combined EC/PR lesions, whereas ablation of the EC alone produced only mild deficits (also see Malkova et al., 2001).

\section{Role of PR circuits in processing complex stimuli}

The present findings are consistent with Burwell's hypothesis (Burwell and Witter, 2002) that deficits associated with PR damage increase with increasing stimulus complexity. More generally, she concluded that deficits associated with PR damage are exacerbated by stimuli of high complexity and/or low discriminability and increased memory load. This could be an apt description of the state of a rat that must differentially respond to various ultrasonic social signals as a function of the immediate environment (Blanchard et al., 1991). Consider the fact that seemingly similar USVs in the $22 \mathrm{kHz}$ range can be reliably elicited as unconditional responses to sex and also as conditional or unconditional responses to threatening or aversive stimuli (Blanchard et al., 1991; Choi and Brown, 2003).

Rats emit a variety of ultrasonic social signals in association with ethologically important interactions (Sales and Pye, 1975; van der Poel and Miczek, 1991), but relatively little is known about how conspecifics normally decode these USVs or discriminate among them. The ability to record and reproduce rat USVs inexpensively and with excellent fidelity now allows these questions to be explored in detail. Studies of both rodents (Young et al., 1997; Bucci et al., 2002; Jarrard et al., 2004) and primates (Suzuki, 1996; Gaffan and Buckley, 1998; Buffalo et al., 1999; Baxter and Murray, 2001) encourage the idea that the PR is importantly involved in processing and/or maintaining representations of complex stimulus configurations. Variations on the approach described here should be useful for exploring the unique or distinctive contributions of PR in fear conditioning to complex cues.

\section{Neurophysiological basis of the CS-dependent $P R$ requirement}

The thalamic relay neurons that project directly to the amygdala are thought to be optimally stimulated by pure tones (Armony et al., 1997). It is, therefore, not surprising that cortical processing is unnecessary for delay conditioning to tone conditional stimuli (Phillips and LeDoux, 1995; Bucci et al., 2000). In contrast, FM signals are most effective in driving neurons in temporal (TE) auditory cortex (Theunissen et al., 2000; Orduna et al., 2001). Specifically, neurons in TE, from which the PR receives its auditory input, appear to be tuned to frequency upsweeps and downsweeps at particular repetition rates (deCharms et al., 1998; Or- duna et al., 2001). If the PR is involved in learning to respond to configurations of elementary features, as hypothesized (Murray and Bussey, 1999), then its input from TE seems to furnish an ideal set of basic feature elements for additional mnemonic processing of these social signals.

Whereas tone cues might be expected to produce the strongest and most consistent sets of afferent inputs to the LA through the subcortical pathway, USV cues should do so through the cortical pathway. TE-PR circuits may be specialized for reliable detection and preferential processing of "prototypes" (Rosch, 1978; Knapp and Anderson, 1984; Rumelhart and McClelland, 1986) of these ultrasonic social signals. One naturally suspects that some neurons in rat TE and/or PR may be tuned to low-frequency $(\sim 1 \mathrm{~Hz})$ repetitions containing upsweeps and/or downsweeps in the $20-30 \mathrm{kHz}$ band, although nothing is known about the stimulus selectivity of these neurons for FM signals in ultrasound.

Others have hypothesized that PR circuits support learning about and/or maintaining abstract or "configural" representations of certain classes of complex stimuli (Murray and Bussey, 1999; Burwell and Witter, 2002). Related ideas have been suggested for hippocampal function (Anagnostaras et al., 2001). The implication is that the impairing effect of preacquisition PR lesions depends on the extent to which these higher-order representations are required for performance. The evidence now suggests that this PR function can subserve both defensive conditioning (Fig. 6B) (Bucci et al., 2000) and working memory (Young et al., 1997; Murray and Richmond, 2001; Jarrard et al., 2004).

\section{References}

Anagnostaras SG, Gale GD, Fanselow MS (2001) Hippocampus and contextual fear conditioning: recent controversies and advances. Hippocampus 11:8-17.

Armony JL, Servan-Schreiber D, Romanski LM, Cohen JD, LeDoux JE (1997) Stimulus generalization of fear responses: effects of auditory cortex lesions in a computational model and in rats. Cereb Cortex 7:157-165.

Baxter MG, Murray EA (2001) Opposite relationship of hippocampal and rhinal cortex damage to delayed nonmatching-to-sample deficits in monkeys. Hippocampus 11:61-71.

Beggs JM, Moyer JR, McGann JP, Brown TH (2000) Prolonged synaptic integration in perirhinal cortical neurons. J Neurophysiol 83:3294-3298.

Blanchard RJ, Blanchard DC (1969) Crouching as an index of fear. J Comp Physiol Psychol 67:370-375.

Blanchard RJ, Blanchard DC, Agullana R, Weiss SM (1991) Twenty-two $\mathrm{kHz}$ alarm cries to presentation of a predator, by laboratory rats living in visible burrow systems. Physiol Behav 50:967-972.

Brown MW, Aggleton JP (2001) Recognition memory: what are the roles of the perirhinal cortex and hippocampus. Nat Rev Neurosci 2:51-61.

Brudzynski SM, Barnabi F (1993) Analysis of $22 \mathrm{kHz}$ ultrasonic vocalization in laboratory rats: long and short calls. Physiol Behav 54:215-221.

Bucci DJ, Phillips RG, Burwell RD (2000) Contributions of postrhinal and perirhinal cortex to contextual information processing. Behav Neurosci 114:882-894.

Bucci DJ, Saddoris MP, Burwell RD (2002) Contextual fear discrimination is impaired by damage to the postrhinal or perirhinal cortex. Behav Neurosci 116:479-488.

Buckley MJ, Gaffan D (1998) Perirhinal cortex ablation impairs configural learning and paired-associate learning equally. Neuropsychologia 36:535-545.

Buffalo EA, Ramus SJ, Clark RE, Teng E, Squire LR, Zola SM (1999) Dissociation between the effects of damage to perirhinal cortex and area TE. Learn Mem 6:572-599.

Burwell RD (2001) Borders and cytoarchitecture of the perirhinal and postrhinal cortices in the rat. J Comp Neurol 437:17-41.

Burwell RD, Amaral DG (1998) Perirhinal and postrhinal cortices of the rat: interconnectivity with the entorhinal cortex. J Comp Neurol 391:293321.

Burwell RD, Witter MP (2002) Basic anatomy of the parahippocampal re- 
gion in monkeys and rats. In: The parahippocampal region, organization and role in cognitive functions (Witter MP, Wouterlood FG, eds), pp 35-59. London: Oxford UP.

Burwell RD, Witter MP, Amaral DG (1995) Perirhinal and postrhinal cortices of the rat: a review of the neuroanatomical literature and comparison with findings from the monkey brain. Hippocampus 5:390-408.

Campeau S, Davis M (1995) Involvement of subcortical and cortical afferents to the lateral nucleus of the amygdala in fear conditioning measured with fear-potentiated startle in rats trained concurrently with auditory and visual conditioned stimuli. J Neurosci 15:2312-2327.

Choi J-S, Brown TH (2003) Central amygdala lesions block ultrasonic vocalization and freezing as conditional but not unconditional responses. J Neurosci 23:8713-8721.

Choi J-S, Lindquist DH, Brown TH (2001) Amygdala lesions prevent conditioned enhancement of the rat eyeblink reflex. Behav Neurosci 115:764-775.

Cohen J (1988) Statistical power analysis for the behavioral sciences. Hillsdale, NJ: Erlbaum.

deCharms RC, Blake DT, Merzenich MM (1998) Optimizing sound features for cortical neurons. Science 280:1439-1444.

Fanselow MS (1997) Species-specific defense reactions: retrospect and prospect. In: Learning, motivation, and cognition (Bouton ME, ed), pp 321341. Washington, DC: American Psychological Association.

Faulkner B, Brown TH (1999) Morphology and physiology of neurons in the rat perirhinal-lateral amygdala area. J Comp Neurol 411:613-642.

Furtak S, Moyer Jr JM, Brown TH (2003) Age-related extinction impairment following fear conditioning to an ultrasonic social signal. Soc Neurosci Abstr 29:114.18.

Furtak SC, Powell TC, Moyer Jr JR, Brown TH (2001) Golgi analysis of perirhinal cortex during rat development. Soc Neurosci Abstr 27:696.4.

Gaffan D, Buckley MJ (1998) Learning and transfer of object-reward associations and the role of the perirhinal cortex. Neuropsychologia 36:535-546.

Herzog C, Otto T (1997) Odor-guided fear conditioning in rats: 2. Lesions of the anterior perirhinal cortex disrupt fear conditioning to the explicit conditioned stimulus but not to the training context. Behav Neurosci 111:1265-1272.

Herzog C, Otto T (1998) Contributions of anterior perirhinal cortex to olfactory and contextual fear conditioning. NeuroReport 9:1855-1859.

Holland PC, Bouton ME (1999) Hippocampus and context in classical conditioning. Curr Opin Neurobiol 9:195-202.

Jarrard LE, Davidson TL, Bowring B (2004) Functional differentiation within the medial temporal lobe in the rat. Hippocampus, in press.

Knapp AG, Anderson JA (1984) Theory of categorization based on distributed memory storage. J Exp Psychol Learn Mem Cognit 10:616-637.

Lindquist DH, Brown TH (2004) Amygdalar NMDA receptors control the expression of associative reflex facilitation and three other conditional responses. Behav Neurosci 118:36-52.

Malkova L, Bachevalier J, Mishkin M, Saunders RC (2001) Neurotoxic lesions of perirhinal cortex impair visual recognition memory in rhesus monkeys. NeuroReport 12:1913-1917.

Maren S (1999) Neurotoxic basolateral amygdala lesions impair learning and memory but not the performance of conditional fear in rats. J Neurosci 19:8696-8703.

Maren S, Aharonov G, Fanselow MS (1997) Neurotoxic lesions of the dorsal hippocampus and Pavlovian fear conditioning in rats. Behav Brain Res $88: 261-274$

Mascagni F, McDonald AJ, Coleman JR (1993) Corticoamygdaloid and corticocortical projections of the rat temporal cortex: a Phaseolus vulgaris leucoagglutinin study. Neuroscience 57:697-725.
McGann JP, Moyer Jr JR, Brown TH (2001) Predominance of late-spiking neurons in layer VI of rat perirhinal cortex. J Neurosci 21:4969-4976.

Meunier M, Bachevalier J, Mishkin M, Murray EA (1993) Effects on visual recognition of combined and separate ablations of the entorhinal and perirhinal cortex in rhesus monkeys. J Neurosci 13:5418-5432.

Moyer Jr JR, Brown TH (1998) Methods for whole-cell recording from visually preselected neurons of perirhinal cortex in brain slices from young and aging rats. J Neurosci Methods 86:35-54.

Moyer Jr JR, McNay EC, Brown TH (2002) Three classes of pyramidal neurons in layer $\mathrm{V}$ of rat perirhinal cortex. Hippocampus 12:218-234.

Murray EA, Bussey TJ (1999) Perceptual-mnemonic functions of perirhinal cortex. Trends Cogn Sci 3:142-151.

Murray EA, Richmond BJ (2001) Role of perirhinal cortex in object perception, memory and associations. Curr Opin Neurobiol 11:188-193.

Naber PA, Witter MP, Lopes da Silva FH (1999) Perirhinal cortex input to the hippocampus in the rat: evidence for parallel pathways, both direct and indirect. A combined physiological and anatomical study. Eur J Neurosci 11:4119-4133.

Orduna I, Edurado III M, Gluck MA, Merzenich MM (2001) Spectrotemporal sensitivities in rat auditory cortical neurons. Hear Res 160:47-57.

Otto T, Cousens G, Herzog C (2000) Behavioral and neuropsychological foundations of olfactory fear conditioning. Behav Brain Res 110:119-128.

Paxinos G, Watson C (1998) The rat brain, Ed 4. San Diego: Academic.

Phillips RG, LeDoux JE (1995) Lesions of the fornix but not the entorhinal or perirhinal cortex interfere with contextual fear conditioning. J Neurosci 15:5308-5315.

Romanski LM, LeDoux JE (1992a) Bilateral destruction of neocortical and perirhinal projection targets of the acoustic thalamus does not disrupt auditory fear conditioning. Neurosci Lett 142:228-232.

Romanski LM, LeDoux JE (1992b) Equipotentiality of thalamo-amygdala and thalamo-cortico-amygdala circuits in auditory fear conditioning. J Neurosci 12:4501-4509.

Rosch E (1978) Principles of categorization. In: Cognition and categorization (Rosch E, Lloyd BB, eds). Hillsdale, NJ: Erlbaum.

Rumelhart DE, McClelland JL (1986) Parallel distributed processing: explorations in the microstructure of cognition: foundations. Cambridge, MA: MIT.

Sales G, Pye D (1975) Ultrasonic communication by animals. London: Chapman and Hall.

Selden NR, Everitt BJ, Jarrard LE, Robbins TW (1991) Complementary roles for the amygdala and hippocampus in aversive conditioning to explicit and contextual cues. Neuroscience 42:335-350.

Sewell GD (1970) Ultrasonic signals from rodents. Ultrasonics 8:26-30.

Shi CJ, Cassell MD (1999) Perirhinal cortex projections to the amygdaloid complex and hippocampal formation in the rat. J Comp Neurol 406:299-328.

Suzuki WA (1996) The anatomy, physiology and functions of the perirhinal cortex. Curr Opin Neurobiol 6:179-186.

Theunissen FE, Sen K, Doupe AJ (2000) Spectral-temporal receptive fields of nonlinear auditory neurons obtained using natural sounds. J Neurosci 20:2315-2331.

van der Poel AM, Miczek KA (1991) Long ultrasonic calls in male rats following mating, defeat and aversive stimulation: frequency modulation and bout structure. Behaviour 119:127-142.

White NM, Salinas JA (2003) Mnemonic functions of dorsal striatum and hippocampus in aversive conditioning. Behav Brain Res 142:99-107.

Young BJ, Otto T, Fox GD, Eichenbaum H (1997) Memory representations with the parahippocampal region. J Neurosci 17:5183-5195. 